University of New Orleans

ScholarWorks@UNO

$9-1983$

\title{
Explicit equations for the second Brewster angle of an interface between a transparent and an absorbing medium
}

\author{
R. M.A. Azzam \\ University of New Orleans, razzam@uno.edu
}

Follow this and additional works at: https://scholarworks.uno.edu/ee_facpubs

Part of the Electrical and Electronics Commons, and the Physics Commons

\section{Recommended Citation}

R. M. A. Azzam, "Explicit equations for the second Brewster angle of an interface between a transparent and an absorbing medium," J. Opt. Soc. Am. 73, 1211-1212 (1983)

This Article is brought to you for free and open access by the Department of Electrical Engineering at ScholarWorks@UNO. It has been accepted for inclusion in Electrical Engineering Faculty Publications by an authorized administrator of ScholarWorks@UNO. For more information, please contact scholarworks@uno.edu. 


\title{
Explicit equations for the second Brewster angle of an interface between a transparent and an absorbing medium
}

\author{
R. M. A. Azzam \\ Department of Electrical Engineering, University of New Orleans, Lakefront, New Orleans, Louisiana 70148
}

Received March 12, 1983

The second Brewster angle $\phi_{B 2}$, at which the ratio $\mathcal{R}_{p} / \mathscr{R}_{s}$ of intensity reflectances $\mathcal{R}_{p}$ and $\mathcal{R}_{s}$ for the parallel $(p)$ and the perpendicular $(s)$ polarizations of a dielectric-conductor interface reaches a minimum, is determined by $\operatorname{Im}\left[(u-\epsilon)(u-\bar{\epsilon})^{2} /(u-2 \bar{\epsilon})^{2}\right]=0$, where $\epsilon$ is the complex ratio of dielectric constants of the media of refraction and incidence, $\bar{\epsilon}=\epsilon /(\epsilon+1)$, and $u=\sin ^{2} \phi_{B 2}$. An equivalent quartic equation in $u$ is also derived that can be solved exactly and explicitly to determine $\phi_{B 2}$ in terms of $\epsilon$.

When monochromatic and collimated totally polarized light (or any other electromagnetic radiation) is reflected at the interface between a transparent and an absorbing medium, the ratio $\mathcal{R}_{p} / \mathcal{R}_{s}$ of the intensity reflectances $\mathcal{R}_{p}$ and $\mathscr{R}_{s}$ for the parallel $(p)$ and perpendicular $(s)$ polarizations reaches a minimum at the so-called second Brewster angle. ${ }^{1}$ Such an angle differs ${ }^{2}$ from the pseudo-Brewster angle of minimum $\mathcal{R}_{p}$, and the principal angle at which the difference $\Delta$ between the reflection phase shifts experienced by the $p$ - and $s$-component vibrations equals $90^{\circ}$.

Whereas explicit equations (cubic in the sine squared of the angle of incidence) exist for the pseudo-Brewster and principal angles, ${ }^{3}$ no similar equation appears to be available for the second Brewster angle, despite a detailed published analysis of the condition of minimum $\mathcal{R}_{p} / \mathcal{R}_{s}$ and its use for determination of optical constants. ${ }^{4}$

The objective of this Letter is to present a relatively simple derivation of explicit equations that determine the second Brewster angle of an interface in terms of the complex relative dielectric constant

$$
\epsilon=\epsilon_{1} / \epsilon_{0}
$$

where $\epsilon_{0}$ is the real dielectric constant of the transparent medium of incidence (medium 0 ) and $\epsilon_{1}$ is the complex dielectric constant of the absorbing medium of refraction. The approach that we follow is similar to that which we have used recently ${ }^{5}$ to determine the pseudo-Brewster angle of an interface in terms of complex $\epsilon$. Its simplicity stems from working with the complex ratio of $p$ and $s$ amplitude-reflection coefficients

$$
\rho=r_{p} / r_{s}=|\rho| \exp (j \Delta)
$$

instead of the real ratio of intensity reflectances

$$
\mathcal{R}_{p} / \mathscr{R}_{s}=|\rho|^{2} \text {. }
$$

The second Brewster angle is determined by the condition that

$$
\frac{\partial}{\partial \phi}|\rho|^{2}=0=2|\rho||\rho|^{\prime} ;
$$

the prime superscript is used throughout to denote the first derivative with respect to the angle of incidence $\phi$. By loga- rithmic differentiation of Eq. (2), we get

$$
\rho^{\prime} / \rho=\left(|\rho|^{\prime} /|\rho|\right)+j \Delta^{\prime}
$$

Because $|\rho| \neq 0$ at any $\phi$, Eqs. (4) and (5) lead to

$$
\operatorname{Re}\left(\rho^{\prime} / \rho\right)=0
$$

as the equation that determines the second Brewster angle.

To proceed from Eq. (6), we write $\rho$ as a function ${ }^{6}$ of $\epsilon$ and $\phi:$

$$
\rho=(1-Y) /(1+Y)
$$

where

$$
Y=\cos \phi\left(\epsilon-\sin ^{2} \phi\right) / \sin ^{2} \phi .
$$

Differentiation of Eqs. (7) and (8) with respect to $\phi$ gives

$$
\begin{aligned}
\rho^{\prime} / \rho & =-2 Y^{\prime} /\left(1-Y^{2}\right) \\
& =\frac{2 \sin \phi\left(1+\cos ^{2} \phi\right) \epsilon-2 \sin ^{3} \phi}{\left(\epsilon-\sin ^{2} \phi\right)^{1 / 2}\left(\sin ^{2} \phi-\epsilon \cos ^{2} \phi\right)} .
\end{aligned}
$$

If we make the convenient change of variable

$$
u=\sin ^{2} \phi
$$

and substitute Eq. (9) into Eq. (6), we obtain

$$
\operatorname{Re}\left[\frac{(\epsilon-u)^{1 / 2}\left(u-\frac{\epsilon}{\epsilon+1}\right)}{\left(u-\frac{2 \epsilon}{\epsilon+1}\right)}\right]=0 .
$$

In reaching Eq. (11), we made use of the fact that, if $\operatorname{Re}(r z)=$ 0 , where $r$ and $z$ are real and complex numbers, respectively, then $\operatorname{Re}(1 / z)=0$.

The square root can be removed from Eq. (11) by noting that, if $\operatorname{Re} z=0$, then $\operatorname{Im} z^{2}=0$; thus

$$
\operatorname{Im}\left[\frac{(u-\epsilon)\left(u-\frac{\epsilon}{\epsilon+1}\right)^{2}}{\left(u-\frac{2 \epsilon}{\epsilon+1}\right)^{2}}\right]=0 .
$$

Equation (12) is, to our knowledge, a new and relatively simple equation that determines the second Brewster angle 


$$
\phi_{B 2}=\sin ^{-1} u^{1 / 2}
$$

for a given complex $\epsilon$ of a given interface.

Equation (12) will always have a solution

$$
0<u<1
$$

irrespective of $\epsilon$. To prove the existence of the second Brewster angle for any given complex $\epsilon$, Eq. (12) can be put in the form $\operatorname{Im}[F(u)]=0$. As $u$ increases from 0 to $1, F(u)$ follows a trajectory in the complex plane that begins at $F(0)$ $=-\epsilon / 4$ and ends at $F(1)=1 /(1-\epsilon)$. From simple geometrical considerations, it becomes apparent that the end points $F(0)$ and $F(1)$ lie on opposite sides of the real axis for any complex $\epsilon$. This guarantees the intersection of the curve of $F(u)$ with the real axis; hence the existence of a solution for Eq. (12), at a certain $u$ in the range of inequality (14).

When $\epsilon$ is real (i.e., for an interface between two transparent media), the second Brewster angle must coincide with the exact Brewster angle of zero parallel reflectance. This is indeed the case because Eq. (11) gives $u=\epsilon / \epsilon+1$, from which $\epsilon=u /(1-u)=\tan ^{2} \phi$, as expected.

To derive from Eq. (12) a more explicit equation for $u$, we first set

$$
\bar{\epsilon}=\epsilon /(\epsilon+1)
$$

and rewrite Eq. (12) in the form

$$
\operatorname{Im}(N / M)=0 \text {, }
$$

where

$$
\begin{aligned}
N & =(u-\epsilon)(u-\bar{\epsilon})^{2} \\
& =u^{3}+\beta_{2} u^{2}+\beta_{1} u+\beta_{0}, \\
M & =(u-2 \bar{\epsilon})^{2} \\
& =u^{2}+\gamma_{1} u+\gamma_{0},
\end{aligned}
$$

and

$$
\begin{gathered}
\beta_{0}=-\epsilon \bar{\epsilon}^{2}, \quad \beta_{1}=\bar{\epsilon}^{2}+2 \epsilon \bar{\epsilon}, \quad \beta_{2}=-\epsilon-2 \bar{\epsilon}, \\
\gamma_{0}=4 \bar{\epsilon}^{2}, \quad \gamma_{1}=-4 \bar{\epsilon} .
\end{gathered}
$$

Next we write

$$
\beta_{k}=\beta_{k r}+j \beta_{k i}, \quad \gamma_{k}=\gamma_{k r}+j \gamma_{k i}, \quad k=1,2,3,
$$

and

$$
N=N_{r}+j N_{i}, \quad M=M_{r}+j M_{i} .
$$

Substitution of Eqs. (20) into Eq. (16) puts the condition for the second Brewster angle in the form

$$
N_{r} M_{i}-N_{i} M_{r}=0 .
$$

Finally, substituting from Eqs. (17), (19), and (20) into Eq. (21), we obtain

$$
a_{4} u^{4}+a_{3} u^{3}+a_{2} u^{2}+a_{1} u+a_{0}=0,
$$

which is a quartic equation with coefficients given by

$$
\begin{aligned}
& a_{0}=\beta_{0 r} \gamma_{0 i}-\beta_{0 i} \gamma_{0 r}, \\
& a_{1}=\beta_{0 r} \gamma_{1 i}+\beta_{1 r} \gamma_{0 i}-\beta_{0 i} \gamma_{1 r}-\beta_{1 i} \gamma_{0 r}, \\
& a_{2}=\beta_{2 r} \gamma_{0 i}+\beta_{1 r} \gamma_{1 i}-\beta_{0 i}-\beta_{1 i} \gamma_{1 r}-\beta_{2 i} \gamma_{0 r}, \\
& a_{3}=\beta_{2 r} \gamma_{1 i}+\gamma_{0 i}-\beta_{1 i}-\beta_{2 i} \gamma_{1 r}, \\
& a_{4}=\gamma_{1 i}-\beta_{2 i} .
\end{aligned}
$$

For a given complex $\epsilon$ ( of a given interface), the coefficients $a_{k}$ can be calculated by use of Eqs. (15), (18), (19), and (23), in that order. Fortunately, a quartic equation can be solved exactly and explicitly ${ }^{7}$ (i.e., without numerical iteration), and, from the root $0<u<1$ of Eq. (22), one determines the second Brewster angle by using Eq. (13).

Before concluding, we note that the condition of maximum minimum parallel reflectance at the pseudo-Brewster angle, discussed in Ref. 5 , has its analog in terms of the reflectance ratio $\mathscr{R}_{p} / \mathscr{R}_{s}=|\rho|^{2}$ and the second Brewster angle. Thus, if $r=|r| e^{j \delta}$ denotes the complex normal-incidence interfacereflection coefficient, we find that, for a given $|r|$, the minimum of $\mathcal{R}_{p} / \mathcal{R}_{s}$ goes to zero at $\delta=0$ and $\delta=180^{\circ}$ and must reach a maximum at some intermediate phase shift $0<\delta<$ $180^{\circ}$. Such $\delta$ is expected to differ from that which leads to maximum minimum $\mathcal{R}_{p}$. A detailed analysis of the condition of maximum minimum $\mathcal{R}_{p} / \mathscr{R}_{s}$ can be pursued along the lines of Ref. 5 .

To summarize, we have derived explicit equations, Eqs. (12) and (22), for the second Brewster angle (at which the ratio of parallel to perpendicular reflectance is minimum) of interfaces between isotropic transparent and absorbing media. Whereas the pseudo-Brewster and principal angles are determined by cubic equations, we have found that the second Brewster angle is determined by a quartic equation, Eq. (22), with the variable of all equations being the sine squared of the incidence angle of interest. Fortunately, quartic equations, like cubic equations, are exactly solvable.

Note added in proof: Measurements on an ultrathin gold foil, kindly provided by Gary Reeves of Los Alamos National Laboratory, confirmed the validity of this technique. These results were presented at the Paris Ellipsometry Conference, June 7-10, 1983.

\section{ACKNOWLEDGMENT}

I am pleased to acknowledge support by the National Science Foundation under grant DMR-8018417.

\section{REFERENCES}

1. J. M. Bennett and H. E. Bennett, in Handbook of Optics, W. G. Driscoll and W. Vaughan, eds. (McGraw-Hill, New York, 1978), pp. 10-11-10-12.

2. H. B. Holl, "Specular reflection and characteristics of reflected light," J. Opt. Soc. Am. 57, 683-690 (1967).

3. S.P.F. Humphreys-Owen, "Comparison of reflection methods for measuring optical constants without polarimetric analysis, and proposal for new methods based on the Brewster angle," Proc. Phys. Soc. London 77, 949-957 (1961).

4. R. F. Potter, "Analytical determination of optical constants based on the polarized reflectance at a dielectric-conductor interface," J. Opt. Soc. Am. 54, 904-906 (1964).

5. R. M. A. Azzam, "Maximum minimum reflectance of parallelpolarized light at interfaces between transparent and absorbing media," J. Opt. Soc. Am. 73, 959-962 (1983).

6. See, for example, R. M. A. Azzam and N. M. Bashara, Ellipsometry and Polarized Light (North-Holland, Amsterdam, 1977), p. 274.

7. See, for example, S. M. Selby, ed., Standard Mathematical Tables, 20th ed. (Chemical Rubber Company, Cleveland, Ohio, 1972), p. 106. 